\section{Are people with schizophrenia more logical than healthy volunteers?}

\author{
GARETH S. OWEN, JOHN CUTTING and ANTHONY S. DAVID
}

\begin{abstract}
Summary Wetested a
phenomenological hypothesis about

theoretical and practical rationality in

people with schizophrenia. This hypothesis

states that in schizophrenia there is an

enhancement of theoretical rationality.

Our case-control experiment supported

this hypothesis. Philosophical models of

rationality that prioritise theoretical over

practical rationality may thereby apply

more in schizophrenic than in healthy

states. The study is an example of how

experimental psychopathology can

illuminate areas of philosophical dispute

that are difficult to settle by thought alone.
\end{abstract}

\section{Declaration of interest None.}

There has been renewed interest in the phenomenological tradition in psychiatry (Owen \& Harland, 2007) which offers a range of hypotheses about different psychopathological states. One notable hypothesis is that schizophrenia is an impairment of commonsense knowing (practical reason), with a preservation - or even accentuation - of systematic cognition (theoretical reason). This concept was made famous by the psychiatrist Minkowski (1927) and can even be found in the anthropological writings of Kant (1798) but has never been experimentally tested. Previous experimental work on rationality in schizophrenia has aimed to explain delusions in schizophrenia in terms of impairments of formal reasoning (Garety \& Hemsley, 1995; Kemp et al, 1997). Results do not generally confirm this model (Cutting, 1997).

In line with the phenomenological hypothesis, we tested whether tasks that are correct from a theoretical (or formal logical) point of view but depart from practical knowledge (common sense) would be performed better by people with schizophrenia than by healthy controls.

\section{METHOD}

Most philosophers conceptualise theoretical rationality as formal logical rationality for which deductive logic is held as the paradigm. Practical rationality or 'common sense', however, has been more difficult to conceptualise. It is generally taken to denote non-formal rationality - a form of knowing that provides the background assumptions about the world that are the basis of shared human practice. It is tacit knowledge within a culture, and includes such things as the pre-theoretical knowledge that the sun rises in the east or that hospitals are buildings. Such knowledge is presupposed and used in everyday practice, and as such becomes something that is separate from theoretical knowledge. The concept of common sense is that there is a form of rationality that is independent of theoretical rationality. The experiment we report assumes these two concepts and takes them to be fundamentally different.

We operationalised theoretical reasoning using syllogisms that were deductively valid or invalid, and common sense using syllogistic content that strongly conformed to or departed from practical knowledge. Two types of syllogism were constructed, in each of which there was a conflict between deductive truth and commonsense truth. The first type was non-commonsense syllogisms that were valid (labelled 'NCS'), for example:

'all buildings speak loudly;

a hospital does not speak loudly;

therefore, a hospital is not a building.'

The second type comprised commonsense syllogisms that were invalid (labelled 'CS'), for example:

"if the sun rises, then the sun is in the east; the sun is in the east; therefore, the sun rises.

Participants were asked by the interviewer (G.O.) to accept the first two sentences of each syllogism as true and then to decide on the truth or falsity of the third sentence. They were told that this rule applied to all the problems and were asked to state it repeatedly until it was clear that they understood it. All participants read the problems aloud. Syllogisms were scored as correct if they were answered logically.

To be more certain that our syllogisms did generate subjective conflict between a logical and a commonsense interpretation in healthy people, we had previously conducted an independent pilot study in which we tested 21 healthy individuals. Verbal reports confirmed the conflict between logical and commonsense interpretations. We discarded three syllogisms that accrued high scores on the basis that their commonsense content was too weak, leaving eight NCS syllogisms and seven CS syllogisms for inclusion in the study reported here.

Ethical approval for the study was gained and all participants gave informed consent. People diagnosed with schizophrenia using standardised criteria (DSMIV; American Psychiatric Association, 1994) and healthy controls were asked to solve the syllogisms in a case-control design. Patients were selected from two innerLondon psychiatric hospitals; the sources were two general in-patient wards and the out-patient and in-patient facilities of a single service specialising in schizophrenia. All participanting patients were taking antipsychotic medication. The control group was selected from a wide variety of informal sources, including acquaintances, porters and staff at several hospitals, and advertisement. Exclusion criteria for both groups were age outside the range 18-65 years; premorbid IQ, estimated using the National Adult Reading Test (Nelson, 1994), outside the range $75-125$ (as at extreme values this measure is a poor guide to full-scale IQ (Russell et al, 2000)); English not native language; other neurological or psychiatric disorder or substance misuse. Medical records were reviewed for all patients and a clinical interview was conducted by a psychiatrist (G.O.) to ensure that criteria were met. Of the 22 patients approached, two were excluded because of elicited histories of epilepsy or heavy substance misuse and three because of NART IQ score $<75$. Of the 21 potential control group members, one was excluded because aged $>65$ years and one because of IQ score $>125$.

Our primary measures were number of syllogisms correct as a total and as subsets according to type (NCS or CS). Potential confounding factors were considered to be IQ, age, gender and years of education. 
GARETH S. OWEN, MRCPsych, JOHN CUTTING, FRCPsych, ANTHONY S. DAVID, FRCPsych, Institute of Psychiatry, London, UK

Correspondence: Department of Psychiatry, PO 68, Institute of Psychiatry, King's College London, De Crespigny Park, London SE5 8AF,UK. Email: g.owen@iop.kcl.ac.uk

(First received 23 February 2007, final revision 18 May 2007, accepted 27 June 2007)

All $t$-tests performed were two-tailed with equal variance not assumed. Using percentage logically correct as the dependent variable, we performed an inter-individual factorial analysis of variance testing for main effects by group (schizophrenia $v$. control) and syllogism type (NCS $v$. CS) and their interaction. Our hypothesis was that the schizophrenia group would outperform the control group.

\section{RESULTS}

Groups were well matched, with 17 patients and 19 controls. There was no significant difference between the groups in premorbid IQ $\quad(t=-0.87, P=0.4), \quad$ age $\quad(t=1.25$, $P=0.22)$ or years of education $(t=-0.06$, $P=0.96)$. About half ( $53 \%$ ) of the control group were men, compared with $65 \%$ of the schizophrenia group.

Table 1 shows the group statistics. As predicted there was a highly significant main effect by group $\left(F_{(1,68)}=8.002\right.$, $P=0.006$ ), with patients outperforming controls. There was also a main effect by syllogism type $\left(F_{(1,68)}=52.916 ; P<0.001\right)$, but no interaction of syllogism type by group $\left(F_{(1,68)}=0.157, P=0.69\right)$. The main effect by syllogism type showed that both groups scored better on the NCS syllogism type than on the CS syllogism type. We take this to be the well-replicated 'belief bias' effect (Evans, 2002), i.e. that logic has a larger effect on unbelievable (NCS) than on believable (CS) conclusions.

In exploratory analysis of the group difference, the effect size using the Cohen's $d$ statistic was 0.82 (large) for the CS syllogism type and 0.54 (medium) for the NCS syllogism type. Similarly, comparisons of means showed significance for the CS syllogism type $(t=-2.37, P=0.026)$ but not for the NCS type $(t=-1.65, P=0.11)$. This suggests that there might be an underlying interaction between syllogism type and group, with the CS syllogism type (commonsense reasoning) accounting for most of the group difference, and that our failure to find it was due to inadequate statistical power.

\section{DISCUSSION}

Our main results show that under conditions where common sense and logic conflict, people with schizophrenia reason more logically than healthy individuals. On a straightforward interpretation this is either because people with schizophrenia are better at logic or because they are worse at common sense. We present some exploratory evidence that it is because they are worse at common sense, but the question remains open.

A few limitations must be mentioned. The number of participants was small, experimental designs using philosophical concepts are novel and case-control studies cannot control for unknown confounding factors. For example, our stimuli did not allow for correct rejections of noncommonsense syllogisms or correct acceptance of commonsense syllogisms.

The results are intriguing because they shed light on reasoning in schizophrenia but also have significance beyond schizophrenia research. They suggest that

Table I Logical responses to syllogisms by patients with schizophrenia compared with a healthy control group

\begin{tabular}{|c|c|c|c|c|}
\hline & \multirow[b]{2}{*}{$n$} & \multirow[b]{2}{*}{ No. of syllogisms } & \multicolumn{2}{|c|}{ Correct responses } \\
\hline & & & Mean (s.d.) & s.e.m. \\
\hline \multicolumn{5}{|l|}{ All syllogisms } \\
\hline Control group & 19 & 15 & $6.21(2.99)$ & 0.69 \\
\hline Schizophrenia group & 17 & 15 & $8.76(3.25)$ & 0.79 \\
\hline \multicolumn{5}{|l|}{ CS syllogisms' } \\
\hline Control group & 19 & 7 & $1.16(1.21)$ & 0.28 \\
\hline Schizophrenia group & 17 & 7 & $2.53(2.01)$ & 0.51 \\
\hline \multicolumn{5}{|l|}{ NCS syllogisms ${ }^{2}$} \\
\hline Control group & 19 & 8 & $5.05(2.46)$ & 0.56 \\
\hline Schizophrenia group & 17 & 8 & $6.24(1.82)$ & 0.44 \\
\hline
\end{tabular}

I. Commonsense syllogisms, invalid.

2. Non-commonsense syllogisms, valid. in situations where commonsense knowledge is at stake, formal norms of rationality are violated by people with schizophrenia to a lesser extent than by healthy individuals. People with schizophrenia seem to have a bias towards theoretical rationality over and above practical rationality. It is an ongoing dispute within philosophy of science whether, as a matter of principle, theoretical reason has priority over practical reason or vice versa (Thagard, 2004). Given that schizophrenia is at its core a pathological state of thinking, our results suggest that concepts of rationality that prioritise theoretical reason over and above practical reason might apply more accurately in a pathological example of human thinking than in a healthy one. This is an example of how experimental psychopathology can shed light on fundamental philosophical debates that have not been settled by argument alone.

\section{REFERENCES}

American Psychiatric Association (1994) Diagnostic and Statistical Manual of Mental Disorders (4th edn) (DSM-IV). APA.

Cutting, J. (1997) Principles of Psychopathology. Oxford University Press.

Evans, J. S. (2002) Logic and human reasoning: an assessment of the deduction paradigm. Psychological Bulletin, 128, 978-996.

Garety, P. \& Hemsley, D. (1995) Delusions:

Investigations into the Psychology of Delusional Reasoning. Maudsley Monographs 36. Oxford University Press.

Kant, I. (1798) Anthropology from a Pragmatic Point of View. Reprinted (1978), trans. V. L. Dowdell. pp. II3I14. Southern Illinois University Press.

Kemp, R., Chua, S., McKenna, P., et al (1997)

Reasoning and delusions. British Journal of Psychiatry, $\mathbf{1 7 0}$ 398-405.

Minkowski, E. (1927) La schizophrenie. (trans. in part by J. Cutting. Reprinted (1986) in The Clinical Roots of the Schizophrenia Concept (eds J. Cutting \& M. Shepherd), pp. 188-212. Cambridge University Press.

Nelson, N. E. (1994) National Adult Reading Test (NART) (2nd edn). nferNelson.

Owen, G. \& Harland, R. (2007) Taking phenomenology seriously. Schizophrenia Bulletin. 33, 105-107.

Russell, A. J., Munro, J., Jones, P. B., et al (2000) The National Adult Reading Test as a measure of premorbid IQ in schizophrenia. British Journal of Clinical Psychology, 39, 297-305.

Thagard, P. (2004) Rationality and science. In Handbook of Rationality (eds) A. Mele \& P. Rawlings, pp. 353-369. Oxford University Press. 